\title{
Acidentes de trabalho com profissionais de saúde de um hospital universitário
}

\author{
Hanne Alves Bakke,*, Nelma Mirian Chagas de Araújo ${ }^{\mathrm{b}}$ \\ a,*h_bakke@yahoo.com.br, UFPB, Brasil \\ bnelmamca@gmail.com, UFPB, Brasil
}

\begin{abstract}
Resumo
Esta pesquisa almejou estudar os registros de acidentes de trabalho envolvendo profissionais de saúde em um hospital universitário, determinando as categorias profissionais com maiores índices de ocorrências e os setores mais críticos da organização. Os dados dos registros de acidentes analisados foram: sexo, idade, profissão, local do acidente, tipo de acidente, agente da lesão, área afetada e número de horas entre o início da jornada e a ocorrência do acidente. Os documentos foram obtidos através da Comissão Interna de Prevenção de Acidentes (CIPA) do hospital e os dados foram analisados com a aplicação do teste do Qui-Quadrado para avaliar as diferenças entre dados. As mulheres e a equipe de enfermagem foram os mais acometidos e a maioria dos acidentes envolveu material biológico. Houve ocorrência significativa entre 0 e 4 horas após o início da jornada. Estas informações deverão embasar medidas preventivas entre os profissionais de saúde e da administração.
\end{abstract}

Palavras-chave

Acidentes de trabalho. Profissionais de saúde. Hospital.

\section{Introdução}

De acordo com o Ministério da Previdência Social, acidente do trabalho é aquele decorrente do exercício do trabalho a serviço da empresa ou do exercício do trabalho dos segurados especiais, podendo ocasionar lesão corporal ou distúrbio funcional, permanente ou temporário, morte e a perda ou a redução da capacidade para o trabalho (BRASIL, 2007). Os acidentes de trabalho dividem-se em três categorias: típicos - aqueles decorrentes da característica da atividade profissional realizada pelo indivíduo; de trajeto - ocorrem durante o percurso entre a residência e o local de trabalho; doenças do trabalho - aqueles ocasionados por qualquer tipo de doença profissional ligada a determinado ramo de atividade.

Os acidentes estão geralmente associados à fatalidade humana, danos materiais, paradas na produção, danos à imagem da empresa, efeitos psicológicos na equipe e perda de produtividade. 0 estudo destas ocorrências permite uma avaliação das relações entre o homem e o ambiente onde ele exerce suas atividades, seu equilíbrio e sua deterioração, aprimorando 0 conhecimento técnico-científico e permitindo o planejamento e a avaliação das ações voltadas para os trabalhadores. Segundo Asfahl (2005), deve-se incluir nesta análise até mesmo acidentes ou incidentes que não tenham culminado em lesões ou doenças, mas que apresentavam potencial para isso, bem como a ocorrência de eventos inesperados e indesejáveis, com o objetivo de que estas situações não ocorram novamente. A análise das causas dos acidentes e a posterior disseminação dessas informações entre os indivíduos expostos às condições perigosas são consideradas procedimentos eficazes de se evitar lesões e doenças.

De acordo com dados do Anuário Estatístico de Acidentes de Trabalho (BRASIL, 2007), a atividade de atendimento hospitalar apresenta, ao longo dos anos 1999 e 2007, uma tendência de crescimento no número de acidentes, totalizando 217.165 registros de acidentes ao longo deste período. A Norma Regulamentadora 4 (Serviços Especializados em Engenharia de Segurança e em Medicina do Trabalho) classifica os serviços médico-hospitalares como organizações que apresentam grau de risco 3 e são considerados como insalubres, expondo pacientes e profissionais a riscos variados. Trata-se 
de um ambiente onde há concentração de pessoas portadoras de várias doenças infectocontagiosas, em que se realizam procedimentos que apresentam riscos de acidentes e doenças para os trabalhadores de saúde e utilizam formas de tratamento que incluem desde equipamentos de alta tecnologia a técnicas rudimentares de assistência, com a aplicação de agentes físicos e químicos com fins terapêuticos (NISHIDE; BENATTI; ALEXANDRE, 2004).

Segundo Rezende (2003), os profissionais que lidam, direta ou indiretamente, com a saúde dos pacientes preocupam-se muito com a assistência oferecida aos usuários, priorizando o seu conforto e bem-estar, e pouco com os riscos inerentes à execução de suas atividades, que podem ser ampliados segundo a diversificação dos processos e organização do trabalho e pela especialidade da assistência. Esses trabalhadores podem sofrer alterações de saúde oriundas da presença da diversidade de agentes e do tempo e da intensidade do contato entre eles e os agentes.

Além disso, o trabalhador lida diariamente com o adoecimento, a miséria, o desemprego, a violência, fatores sociais da produção da doença, tendo em vista a inclusão/exclusão social que se estabelece nesse modo de produção no contexto social (FORTUNA et al., 2002). Estão também acopladas a estes fatores, de acordo com Ruiz, Barboza e Soler (2004), sobrecarga de serviço, salários insuficientes, situação ocupacional insatisfatória e mecanismos formais e informais de controle dos trabalhadores. Martino e Misko (2004) citam ainda a exposição a situações de elevada tensão emocional, associadas a longas jornadas de trabalho, condições de insalubridade do ambiente laboral, baixos salários e duplo emprego.

Acidentes ocupacionais que envolvem trabalhadores hospitalares têm grande impacto econômico devido à perda de mão-de-obra qualificada pelas lesões ocupacionais, bem como pelo dano irreparável à imagem da instituição hospitalar, quando profissionais infectam os pacientes. Segundo Van Gemert-Pijnen et al. (2006), essas organizações têm a obrigação de informar e treinar a equipe para que estes sigam as medidas cabíveis para manter a segurança nas organizações.

Desta forma, este trabalho teve como objetivo estudar o histórico de acidentes de trabalho em um hospital universitário, levantando quais os profissionais de saúde mais acometidos, os setores mais críticos na organização quanto à ocorrência de acidentes e quais as suas causas.

\section{Metodologia}

Trata-se de um estudo documental, com uma abordagem qualiquantitativa. A pesquisa foi realizada em hospital universitário de uma capital do país e utilizou o documento de registro de acidentes de trabalho, cujo histórico foi disponibilizado pela Comissão Interna de Prevenção de Acidentes (CIPA). À CIPA cabe a notificação dos acidentes de trabalho ocorridos com todos os funcionários do hospital. Esta comissão passou, recentemente, por eleições e não possui uma sede fixa no hospital. Os integrantes ressaltaram, ainda, que a organização não tem um sistema de gerenciamento de riscos ou de acidentes de trabalho.

Os registros analisados foram aqueles que envolviam profissionais de saúde (equipes de enfermagem, médica e de fisioterapia). Para isso, foi elaborada uma tabela para o levantamento das informações acerca dos acidentes ocorridos, cujas colunas correspondiam a: sexo, idade, profissão, local do acidente, tipo de acidente, agente da lesão, área afetada e número de horas entre o início da jornada de trabalho e a ocorrência do acidente. Os dados foram analisados através de estatística descritiva (percentuais, média, desvio padrão) e do teste de Qui-Quadrado $(\alpha<0,1)$, para avaliar a significância da diferença entre os dados.

\section{Resultados e discussão}

Entre 2001 e 2008 foram registrados no hospital 38 acidentes de trabalho, desses, 34 ocorrências envolviam profissionais de saúde. 0 ano de 2005 foi o de maior quantidade de notificações (10), seguido dos anos de 2006 e 2007, com oito ocorrências cada, e 2004, com cinco ocorrências. Os anos 2001, 2003 e 2008 apresentaram apenas uma notificação cada e, em 2002, há ausência de registros.

Ao realizar uma comparação do número de registros de acidentes de trabalho neste hospital com pesquisas realizadas em outras instituições de outros estados, percebe-se número inferior de notificações. Em um hospital no Estado de São Paulo foram registrados, no ano de 1998, 398 acidentes de trabalho (CANINI et al., 2002). Na pesquisa realizada por Ruiz, Barboza e Soler (2004), em um hospital geral de ensino com 2.800 trabalhadores em São Paulo foram registrados, entre os anos de 2000 e 2001, um total de 861 ocorrências. Já em um hospital público no Rio de Janeiro foram 265 registros de acidentes entre os anos de 1997 e 1998 (OSÓRIO; MACHADO; MINAYO-GOMEZ, 2005). Outro estudo, também em um hospital universitário em São Paulo, identificou 53 acidentes apenas 
com exposição a líquidos corporais humanos no período de um ano (BALSAMO; FELLI, 2006). A quantidade de registros de acidentes de trabalho encontrada no hospital estudado, à luz de outras pesquisas em instituições semelhantes, levanta a suspeita de alto índice de subnotificação dos acidentes ocorridos, tendo em vista o número de trabalhadores na instituição e a quantidade de atividades e procedimentos realizados.

Conformeabordadonaliteratura, asubnotificação dos acidentes de trabalho na área hospitalar é preocupante. Segundo Napoleão et al. (2000), esta é decorrente principalmente da avaliação do profissional de que a situação ou lesão ocorrida não é de risco. No entanto, outras causas, tais como o desconhecimento da obrigatoriedade da notificação do acidente, a falta de tempo devido ao excessivo ritmo de trabalho ou até mesmo o medo de demissão, são citadas entre os profissionais acidentados. Neste estudo, levanta-se também a possível influência da ausência de uma sede fixa da comissão para registro das ocorrências.

A notificação correta e a apuração adequada dos fatos relacionados aos acidentes de trabalho são de extrema importância, pois permitem aos gestores do hospital conhecer as situações de riscos e intervir com medidas e ações direcionadas, mitigando os efeitos econômicos e sociais dos acidentes de trabalho nestas instituições.

Através dos registros de acidentes do hospital, observou-se que a notificação do acidente de trabalho entre 2001 e 2008 ocorreu no mesmo dia em 16 casos, o que representa $47 \%$ do total (Tabela 1). A demora em notificar o acidente de trabalho, especialmente em se tratando da exposição a riscos biológicos, pode definir a eficácia do tratamento pós-exposição, que deve tentar reduzir o tempo entre o momento do acidente e do atendimento.

Dos 34 acidentes, 33 são típicos e um é de trajeto. Houve ocorrências em maior quantidade de pessoas do sexo feminino (29 ocorrências), bem como entre indivíduos casados (19). A idade dos indivíduos acidentados varia entre 21 e 55 anos (média de 36,03 anos e desvio padrão de 11,12), onde a maioria dos trabalhadores tinha entre $21 \mathrm{e}$ 40 anos $(64,7 \%)$ - Tabela 2.

Os valores quanto a sexo e estado civil corroboram os dados encontrados na pesquisa de Ruiz et al. (2004), em que as mulheres foram acometidas em 75,4\% dos casos, indivíduos casados em $40 \%$, e a faixa etária de maior incidência foi de 31 a 40 anos.
A quantidade de registros entre os profissionais de enfermagem (auxiliar, técnico e enfermeiro), somada, equivale a 73,6\% das ocorrências (Figura 1). Este fato reforça a preocupação evidenciada pela vasta literatura sobre acidentes de trabalho entre profissionais desta área por estarem mais expostos a riscos ocupacionais devido às peculiaridades das atividades de cuidados diretos e ininterruptos aos pacientes (ALEXANDRE; BENATTI, 1998; SANTOS, 2001; CANINI et al., 2002; BARBOZA; SOLER, 2003; XELEGATI; ROBAZZI, 2003; NISHIDE; BENATTI; ALEXANDRE, 2004; XELEGATI et al., 2006).

Todavia, percebe-semaiorquantidadederegistros entre auxiliares de enfermagem (15 ocorrências), que correspondem a $44,1 \%$ do total de acidentes ocorridos. Segundo Ruiz, Barboza e Soler (2004), o maior envolvimento de auxiliares de enfermagem nas ocorrências é compreensível, tendo em vista o alto contingente destes trabalhadores nas equipes e as atividades que lhes são atribuídas pelos enfermeiros, que as coordenam. Neste estudo, esta categoria apresentou mais de $90 \%$ das notificações de acidentes de trabalho entre os profissionais da equipe de enfermagem. Há outros estudos que comprovam a maior incidência de acidentes de trabalho nesta classe (CANINI et al., 2002; REZENDE, 2003; NISHIDE; BENATTI, 2004; NISHIDE; BENATTI; ALEXANDRE, 2004; BALSAMO e FELLI, 2006; CASSOLI, 2006; MACHADO, 2006), que também é a categoria mais acometida pelo afastamento do trabalho (BARBOZA; SOLER, 2003), representando $82,3 \%$ do total.

Tabela 1. Número de dias entre o acidente de trabalho no hospital e a notificação (2001-2008).

\begin{tabular}{ccc}
\hline Número de dias & Número de registros & $\%$ \\
\hline 0 & 16 & 47,0 \\
1 & 9 & 26,5 \\
2 & 4 & 11,8 \\
Acima de 2 & 2 & 5,9 \\
Não identificado & 3 & 8,8 \\
Total & 34 & 100 \\
\hline
\end{tabular}

Tabela 2. Distribuição dos acidentes de trabalho por faixa etária, no período entre 2001 e 2008.

\begin{tabular}{ccc}
\hline Faixa etária & Número & $\%$ \\
\hline 21 a 30 & 11 & 32,35 \\
31 a 40 & 11 & 32,35 \\
41 a 50 & 6 & 17,65 \\
51 a 60 & 6 & 17,65 \\
Total & 34 & 100 \\
\hline
\end{tabular}




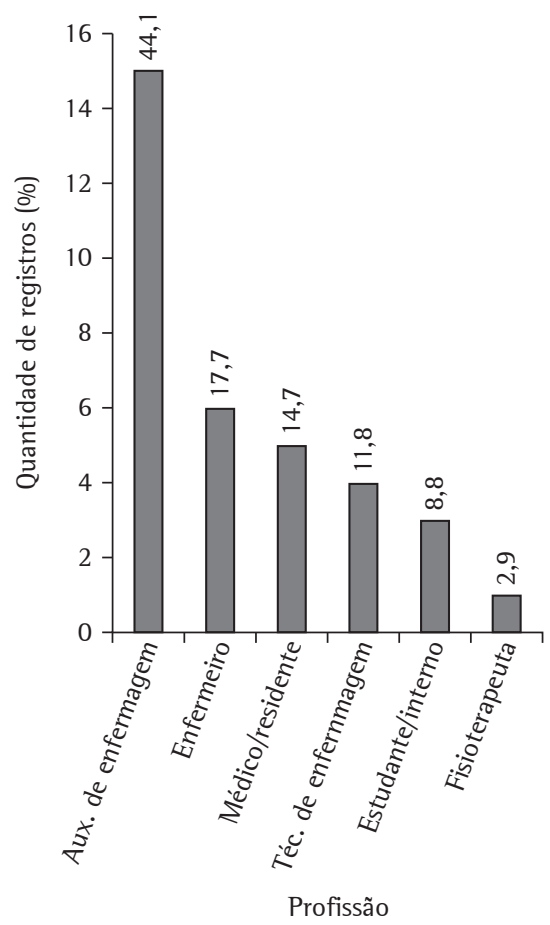

Figura 1. Quantidade de registros de acidentes de trabalho por profissão (2001-2008).

Destacam-se, também, os registros de acidentes dentre estudantes da área de saúde. A sua ocorrência e notificação despertam para a necessidade de políticas de segurança e saúde ocupacional desde a formação do profissional, onde as práticas que exercerão no decorrer de sua vida laboral sejam repassadas e aprendidas.

0 risco ao qual um profissional de saúde está exposto é inerente à sua atividade e à complexidade da assistência. É provável que alguns setores do hospital apresentem mais riscos que outros, variando de acordo com as atividades desempenhadas pelos trabalhadores e com a gravidade dos pacientes e das patologias neles tratadas. Balsamo e Felli (2006) detectaram a unidade de emergência como o local com maior percentual de acidentes de trabalho $(16,67 \%)$, seguido da Clínica Médica e da UTl (com 12,5\%, cada). Em um estudo realizado em três países africanos acerca de exposição acidental a sangue entre profissionais da área de saúde, detectou-se o departamento de medicina como principal fonte deste tipo de acidente, com 205 ocorrências, seguido do setor de ginecologia e obstetrícia (158), cirurgia (102), radiologia (54), UTI (28), cirurgia (6), emergência (6), laboratório (3) e clínica (2) (TARANTOLA et al., 2005). Já Cassoli (2006) identificou, dentre os diversos setores do hospital, o ambiente cirúrgico, composto pelo ambulatório, enfermaria e centro cirúrgico, como principal local de ocorrências de acidentes de trabalho, totalizando 285 ocorrências. A clínica médica apresentou 291 acidentes, a UTl, 155, o pronto socorro, 112, o laboratório, 48, o setor de odontologia, 31 e o berçário, 16.

Em relação ao hospital estudado, a análise do histórico de registros de acidentes constatou maior ocorrência de acidentes no Centro de Terapia Intensiva (CTI), sete registros, quando comparado aos demais setores do hospital (Figura 2). Embora a diferença não seja significativa para o teste de Qui-Quadrado, é um setor crítico, tendo em vista o número de ocorrências neste hospital e a presença deste, em outros estudos, como fonte de grande quantidade de acidentes. Além disso, o CTI, no estudo de Barboza e Soler (2003), foi o local que apresentou a segunda maior ocorrência de afastamentos do trabalho de profissionais de enfermagem.

0 risco biológico é o que se apresenta com maior intensidade entre os profissionais que prestam assistência à saúde. Este fato foi constatado nesta pesquisa: os acidentes que envolveram material biológico (material pérfuro-cortante e fluídos corporais) corresponderam a 76\% das ocorrências neste hospital (Figura 3). Em um hospital em São Paulo, os acidentes de trabalho com material biológico envolvendo material pérfuro-cortante e fluídos corporais representaram 50,9\% das notificações (RUIZ; BARBOZA; SOLER, 2004).

A Figura 3 evidencia, também, que o material pérfuro-cortante ocasionou mais da metade dos acidentes de trabalho entre os profissionais de saúde do hospital estudado, totalizando 22 notificações. Os acidentes envolvendo este tipo de material não se referem apenas à manipulação destes objetos para a realização de intervenção terapêutica, mas também a sua manipulação e descarte e incluem ocorrências com agulhas e peças cirúrgicas. Este comportamento foi semelhante ao detectado por outras pesquisas sobre acidentes de trabalho em hospitais: a pesquisa de Ruiz, Barboza e Soler (2004) identificou como principal fonte da ocorrência de acidentes o material pérfuro-cortante, que representou 40,4\% do total de acidentes no hospital (348 notificações); no estudo de Cassoli (2006), houve 875 ocorrências, o que equivaleu a 80,2\% das exposições a material biológico; no Estado do Rio de Janeiro, durante os anos 1997 a 2004, foram reportados 15.035 acidentes com material biológico nos hospitais da cidade, - aproximadamente 90\% das exposições foram percutâneas e ocorreram devido ao re-encapeamento de agulhas, à realização de procedimentos cirúrgicos e ao manuseio de 


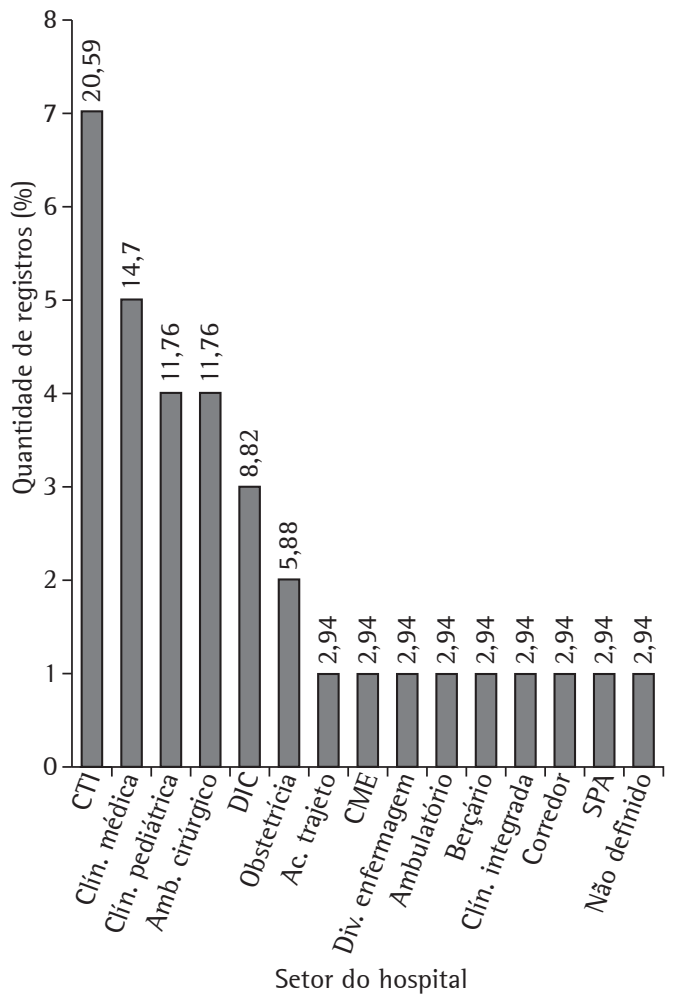

Figura 2. Quantidade de registros de acidentes de trabalho entre os profissionais de saúde do hospital (2001-2008). Nota: SPA - Serviço de Pronto-Atendimento; CME - Centro de Material de Esterilização; DIC - Doenças Infectocontagiosas; CT1 - Centro de Terapia Intensiva.

equipamentoe, à manipulação do lixo, durante o descarte do material pérfuro-cortante em recipientes adequados e com punção venosa percutânea (RAPPARINl et al., 2007). Segundo Marziale, Nishimura e Ferreira (2004), os acidentes com material pérfuro-cortante infectados com sangue são responsáveis por 80 a $90 \%$ das transmissões de doenças infecciosas entre trabalhadores de saúde.

Um fator interessante que foi constatado através da descrição do acidente em dois casos foi a ocorrência do mesmo durante o re-encapeamento da agulha e a desconexão manual da agulha após uso no paciente, procedimento que vedado pela Norma Regulamentadora (NR) 32. Portanto, percebe-se que, neste hospital, é necessária uma melhor conscientização dos profissionais que lidam com o material pérfuro-cortante, visto que a prevenção deste tipo de acidente é uma medida profilática simples.

Apesar de apenas uma ocorrência, chama a atenção o acidente envolvendo uma mordida desferida pelo paciente ao profissional de assistência à saúde. Em um estudo realizado em hospital geral

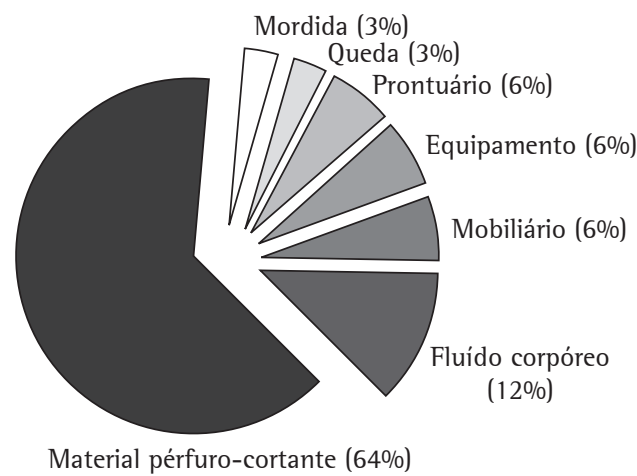

Figura 3. Agentes de lesão nos acidentes de trabalho em profissionais de saúde (2001-2008).

durante o período de 2000 a 2001, houve 22 relatos de agressões físicas sofridas pelos profissionais de saúde, representando 2,6\% do total de acidentes na instituição (RUIZ; BARBOZA; SOLER, 2004). Já Nishide e Benatti (2004), quando perguntaram aos profissionais de saúde de uma UTI acerca dos riscos ocupacionais envolvidos em sua atividade, obtiveram como resposta a agressividade dos pacientes, citada por três indivíduos, equivalendo a 4\% das respostas. Cezar e Marziale (2006) relatam que 100\% dos enfermeiros, $88 \%$ dos técnicos e auxiliares e $85 \%$ dos médicos e um setor de urgência em Londrina foram vítimas de violência ocupacional: agressões verbais, assédio moral e verbal, agressões físicas, competição entre colegas, roubos e maus tratos. Estas ocorrências ratificam estudos que apontam o paciente, bem como os colegas de trabalho, como fontes diretas de riscos à integridade física destes profissionais, mesmo quando o objetivo principal da assistência à saúde seja a recuperação e o bem-estar do paciente.

Os acidentes causados por equipamentos decorrem da manipulação de máquinas de assistência à saúde, tais como bombas de infusão e ventilador mecânico. Aqueles que foram enquadrados na categoria mobiliário resultaram de acidentes com ventiladores de ar e longarinas. Houve também um acidente de trajeto que resultou em uma queda e duas ocorrências envolvendo o prontuário médico.

A realização de atividades que exigem destreza e precisão pelos profissionais de saúde os deixa mais vulneráveis a lesionar os membros superiores, especialmente dedos e mãos. Este fato foi confirmado, visto que houve 28 ocorrências afetando esta parte do corpo - dedos das mãos, mãos e antebraço - (Figura 4), comportamento que corrobora a literatura existente sobre o assunto (RUIZ; BARBOZA; SOLER, 2004; CASSOLI, 2006; MACHADO, 2006). 


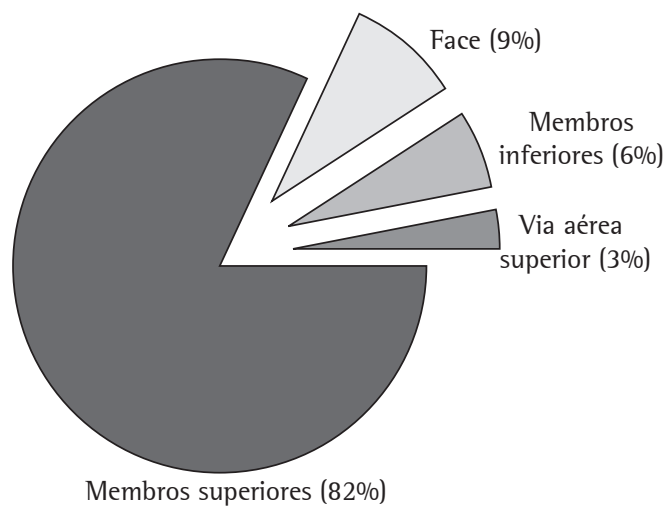

Figura 4. Regiões do corpo afetadas nos acidentes de trabalho de profissionais de saúde no hospital (2001-2008).

As notificações envolvendo a face são advindas da exposição de mucosas dos profissionais (região oral ou ocular) ao respingo de secreções dos pacientes. Os membros inferiores foram lesionados em quedas, no caso do acidente de trajeto sofrido pelo profissional, e pelo trauma ocasionado pela queda de um prontuário. A notificação envolvendo a via aérea superior foi decorrente da aspiração do ar remanescente do tubo oro-traqueal utilizado por um paciente.

No estudo de Ruiz, Barboza e Soler (2004), a região da cabeça e do pescoço foi afetada em 14\% dos acidentes notificados e os membros inferiores, a segunda região do corpo mais afetada, em 15,3\% do total. Na pesquisa de Cassoli (2006) foi identificado que as mucosas foram atingidas em $158(14,5 \%)$ acidentes e os membros inferiores em 2,9\% dos casos em um hospital em São Paulo.

Outro fator a ser estudado é o turno de ocorrência dos acidentes de trabalho entre os profissionais de saúde. Os horários dos turnos foram definidos obedecendo aos horários das escalas dos profissionais: matutino, entre 7 e 13 horas, vesperal, entre 13 e 19 horas, e noturno, entre 19 e 7 horas sendo, portanto, duas vezes mais longo do que cada um dos anteriores. 0 turno da manhã teve relativamente $(\alpha=33,7 \%$ pelo teste do Qui-Quadrado) maior número de ocorrências de acidentes de trabalho, seguido do noturno e do da tarde (Tabela 3). Isto pode estar relacionado a maior intensidade do ritmo de trabalho neste período, pois há mais procedimentos terapêuticos de administração de medicamentos e de coleta de material para exames pela manhã do que em outros turnos (BRAGA, 2000; RUIZ; BARBOZA; SOLER, 2004).

Estes resultados devem ser interpretados com cuidado por diversos fatores: (1) a desigualdade nos intervalos dos turnos da manhã, tarde e noite, (2) a diferença no número de procedimentos realizados por turno (3) e a relação entre o número de procedimentos por profissional envolvido, dentre outros. Para cada fator, deslumbram-se correções nos dados coletados, apesar de não sugeridas na literatura, sendo necessários estudos mais aprofundados para sua validação.

No primeiro caso, almejando-se manter uma igualdade nos intervalos, o turno da noite deveria ser dividido em duas partes iguais, com cinco ocorrências em cada. No entanto, esta correção desconsidera a diferença no ritmo de trabalho entre turnos e, consequentemente, a exposição a diferentes níveis de risco.

No segundo caso, pode-se considerar o percentual do número de procedimentos realizados em cada período. A título de exemplo numérico, supondo-se que $60 \%$ dos procedimentos são realizados pela manhã, $20 \%$ à tarde e $10 \%$ à noite, através da divisão destes percentuais pelos respectivos turnos, obtém-se 25 acidentes pela manhã, 40 à tarde e 110 à noite. Isso significa dizer que, se o mesmo número de procedimentos fosse realizado em cada turno, provavelmente o noturno apresentaria maior quantidade de acidentes, dada a necessidade de vigília com relação ao estado de saúde dos pacientes. Esse resultado demonstra uma ordem de importância inversa da apresentada com a análise dos dados absolutos e corrobora estudos que tratam da influência da privação do sono e do ritmo circadiano no desempenho de trabalhadores noturnos e nas alterações no estado de humor (GASPAR; MORENO; MENNA-BARRETO, 1998; GONÇALVES, 2002; IIDA, 2005).

Para estudar o número de horas entre o início da jornada de trabalho e o acidente foram criadas quatro categorias: de 0 até 4 horas, de 4 até 8 horas, de 8 até 12 horas e acima de 12 horas. 0 intervalo de 0 até 4 horas de serviço teve significativamente maior número de ocorrências de acidentes ( $\alpha=0,001$ pelo teste do Qui-Quadrado), indicando o início da jornada de trabalho como horário mais crítico para a ocorrência de acidentes (Tabela 4). Estes dados corroboram aqueles encontrados por Nishide, Benatti e Alexandre (2004), sendo atribuídos às atividades a serem desempenhadas pelos profissionais no início do plantão, tais como:

Tabela 3. Número de acidentes de trabalho por turno (20012008).

\begin{tabular}{cc}
\hline Turno do acidente & Número de acidentes \\
\hline Matutino (7-13 horas) & 15 \\
Vesperal (13-19 horas) & 8 \\
Noturno (19-07 horas) & 11 \\
\hline
\end{tabular}


Tabela 4. Horas entre o início da jornada de trabalho e o acidente (2001-2008).

\begin{tabular}{cc}
\hline Horas de serviço & Número de acidentes \\
\hline 0 até 4 horas & 17 \\
4 até 8 horas & 7 \\
8 até 12 horas & 9 \\
acima de 12 horas & 1 \\
\hline
\end{tabular}

coleta de sangue para exames laboratoriais e preparo e administração de algumas drogas.

Os registros de acidentes de trabalho do hospital em estudo não possuíam dados referentes a afastamentos ou morte ou o Código Internacional de Doença (CID), inviabilizando a análise destes. Portanto, é necessária a conscientização da CIPA quanto à importância destes dados para o acompanhamento dos trabalhadores e da gravidade do acidente, bem como para analisar o impacto sobre a produtividade e a produção decorrentes dos acidentes de trabalho.

\section{Conclusão}

A análise do histórico de acidentes de trabalho do hospital estudado levanta suspeita de subnotificação, tendo em vista que entre os anos de 2001 e 2008 foram notificados apenas 34 ocorrências envolvendo profissionais de saúde. Alguns resultados encontrados corroboram a literatura, tais como: maior incidência de acidentes de trabalho entre os profissionais de enfermagem (auxiliares e técnicos de enfermagem e enfermeiros) e predominância de acidentes com material pérfurocortante. Os acidentes de trabalho ocorreram, com diferença significativa, entre 0 e 4 horas do início da jornada de trabalho. Além disso, pôde-se constatar que, no hospital estudado, o Centro de Terapia Intensiva apresentou, relativamente, maior quantidade de acidentes de trabalho.

Considerando os diversos agentes aos quais os profissionais de saúde estão expostos e as suas repercussões na saúde dos trabalhadores e na organização, percebe-se a relevância de um sistema que priorize os trabalhadores destes ambientes. A implantação de um Sistema de Gestão de Segurança e Saúde Ocupacional auxilia no reconhecimento e análise dos riscos ocupacionais nestes ambientes, com o objetivo de evitar ou minimizar a ocorrência de acidentes de trabalho, gerenciá-los, embasar as tomadas de decisões, as medidas de controle e de prevenção, e a promoção da conscientização sobre práticas seguras entre os profissionais. 0 cuidado com a gestão da Segurança e Saúde Ocupacional, além de possibilitar a redução nos gastos na instituição, pode melhorar a imagem da mesma diante de seus usuários e motivar seus funcionários, proporcionando maiores lucros e produtividade e melhor qualidade de vida no trabalho.

Portanto, o estudo minucioso acerca das causas dos acidentes de trabalho pode contribuir significativamente para a redução de sua ocorrência. 0 trabalhador, quando orientado, pode evitar as situações de risco e a administração pode direcionar e adaptar medidas mitigatórias de risco à realidade desses profissionais.

\section{Referências}

ALEXANDRE, N. M. C.; BENATTI, M. C. C. Acidentes de trabalho afetando a coluna vertebral: um estudo realizado com trabalhadores de enfermagem de um hospital universitário. Revista Latino-Americana de Enfermagem, v. 6, n. 2, p. 65-72, 1998.

ASFAHL, C. R. Gestão de segurança do trabalho e de saúde ocupacional. São Paulo: Reichmann e Autores Editores, 2005.

BALSAMO, A. C.; FELLl, V. E. A. Estudo sobre os acidentes de trabalho com exposição aos líquidos corporais humanos em trabalhadores da saúde de um hospital universitário. Revista Latino-Americana de Enfermagem, v. 14, n. 3, p. 346-353, 2006.

BARBOZA, D. B.; SOLER, Z. A. S. G. Afastamentos do trabalho na enfermagem: ocorrências com trabalhadores de um hospital de ensino. Revista Latino-Americana de Enfermagem, v. 11, n. 2, p. 177-183, 2003.

BRAGA, D. Acidente de trabalho com material biológico em trabalhadores da equipe de enfermagem do centro de pesquisas Hospital Evandro Chagas. um olhar da saúde do trabalhador. 2000. 86 f. Dissertação (Mestrado em Saúde do Trabalhador e Ecologia Humana) - Escola Nacional de Saúde Pública, Centro de Estudos de Saúde do Trabalhador e Ecologia Humana, Fundação Oswaldo Cruz, Rio de Janeiro.

BRASIL. Ministério da Previdência Social. Anuário estatístico da Previdência Social 2007. Brasília, 2007. Disponível em: <www.mpas.gov.br>. Acesso em: 05 mar. 2008.

CANINI, S. R. M. S. et al. Acidentes perfurocortantes entre trabalhadores de enfermagem de um hospital universitário do interior paulista. Revista Latino-Americana de Enfermagem, v. 10, n. 2, p. 172-178, 2002.

CASSOLl, L. M. Acidente ocupacional com material biológico: adesão ao seguimento ambulatorial segundo as características do acidente e do acidentado. 2006. 136 f. Dissertação (Mestrado em Moléstias Infecciosas e Parasitárias) - Departamento de Moléstias Infecciosas e Parasitárias, Universidade de São Paulo, São Paulo. Disponível em: <http://www.teses.usp.br/teses/ disponiveis/5/5134/tde-31052007-121005/ >. Acesso em: 22 out. 2007.

CEZAR, E. S.; MARZIALE, M. H. P. Problemas de violência ocupacional em um serviço de urgência hospitalar da Cidade de Londrina, Paraná, Brasil. Cadernos de Saúde Pública, v. 22, n. 1, p. 217-221, 2006.

FORTUNA, C. M. et al. Alguns aspectos do trabalho em saúde: os trabalhadores e os processos de gestão. Saúde em Debate: Revista do Centro Brasileiro de Estudos de Saúde, v. 26, n. 62, p. 272-281, 2002. 
GASPAR, S.; MORENO, C.; MENNA-BARRETO, L. Os plantões médicos, o sono e a ritmicidade biológica. Revista da Associação Médica Brasileira, v. 44, n. 3, p. 239-245, 1998.

GONÇALVES, N. M. A. Estudo das falhas humanas da equipe de enfermagem no plano assistencial. 2002. $138 \mathrm{f}$. Dissertação (Mestrado em Engenharia de Produção) Universidade Federal de Santa Catarina, Florianópolis.

IIDA, 1. Ergonomia - projeto e produção. 2 ed. São Paulo: Edgard Blucher, 2005.

MACHADO, A. A. Fatores relacionados à adesão de trabalhadores da área de saúde que sofreram acidente ocupacional com risco biológico. 2006. 197 f. Tese (Livre Docência) - Faculdade de Medicina de Ribeirão Preto, Universidade de São Paulo, Ribeirão Preto.

MARTINO, M. M. F.; MISKO, M. D. Estados emocionais de enfermeiros no desempenho profissional em unidades críticas. Revista da Escola de Enfermagem - USP, v. 38, n. 2, p. 161-167, 2004.

MARZIALE, M. H. P.; NISHIMURA, K. Y. N.; FERREIRA, M. M. Riscos de contaminação ocasionados por acidentes de trabalho com material pérfuro-cortante entre trabalhadores de enfermagem. Revista Latino-Americana de Enfermagem, v. 12, n. 1, p. 36-42, 2004.

NAPOLEÃO, A. A. et al. Notas e informações: causas de subnotificação de acidentes do trabalho entre trabalhadores de enfermagem. Revista Latino-Americana de Enfermagem, v. 8, n. 3, p. 119-120, 2000.

NISHIDE, V. M.; BENATTI, M. C. C. Riscos ocupacionais entre trabalhadores de enfermagem em uma Unidade de Terapia Intensiva. Revista da Escola de Enfermagem USP, v. 38, n. 4, p. 406-414, 2004.

NISHIDE, V. M.; BENATTI, M. C. C.; ALEXANDRE, N. M. C. Ocorrência de acidente do trabalho em uma Unidade de Terapia Intensiva. Revista Latino-Americana de Enfermagem, v. 12, n. 2, p. 204-211, 2004.

OSÓRIO, C.; MACHADO, J. M. H.; MINAYO-GOMEZ, C. Proposição de um método de análise coletiva dos acidentes de trabalho no hospital. Cadernos de Saúde Pública, v. 21, n. 2, p. 517-524, 2005.
RAPPARINI, C. et al. Occupational exposures to bloodborne pathogens among healthcare workers in Rio de Janeiro, Brazil. Journal of Hospital Infection, v. 65, p. 131-137, 2007. Disponível em: <linkinghub.elsevier. com/retrieve/pii/S0195670106004695>. Acesso em: 07 nov. 2007.

REZENDE, M. P. Agravos à saúde de auxiliares de enfermagem resultantes da exposição ocupacional aos riscos físicos. 2003. 127 f. Dissertação (Mestrado em Enfermagem Fundamental) - Universidade de São Paulo, Ribeirão Preto.

RUIZ, M. T.; BARBOZA, D. B.; SOLER, Z. A. S. G. Acidentes de trabalho: um estudo sobre esta ocorrência em um hospital geral. Revista Arquivos de Ciências da Saúde, v. 11 , n. 4, p. 219-224, 2004

SANTOS, P. R. Estudo do processo de trabalho da enfermagem em hemodinâmica: cargas de trabalho e fatores de riscos à saúde do trabalhador. 2001. 145 f. Dissertação (Mestrado em Saúde do Trabalhador e Ecologia Humana) - Fundação Oswaldo Cruz, Rio de Janeiro.

TARANTOLA, A. et al. A descriptive, retrospective study of 567 accidental blood exposures in healthcare workers in three West African countries. Journal of Hospital Infection, v. 60, p. 276-282, 2005. Disponível em: <http://linkinghub. elsevier.com/retrieve/pii/S0195670105000381>. Acesso em: 07 nov. 2007.

VAN GEMERT-PIJNEN, J. et al. Effectiveness of protocols for preventing occupational exposure to blood and body fluids in Dutch hospitals. Journal of Hospital Infection, v. 62, p. 166-173, 2006. Disponível em: linkinghub.elsevier. com/retrieve/pii/S0195670105003099>. Acesso em: 07 nov. 2007.

XELEGATI, R.; ROBAZZl, M. L. C. C. Riscos químicos a que estão submetidos os trabalhadores de enfermagem: uma revisão de literatura. Revista Latino-Americana de Enfermagem, v. 11, n. 3, p. 350-356, 2003.

XELEGATl, R. et al. Riscos ocupacionais químicos identificados por enfermeiros que trabalham em ambiente hospitalar. Revista Latino-Americana de Enfermagem Online, v. 14, n. 2, 2006. Disponível em: <www.scielo.br/pdf/rlae/ v14n2/pt_v14n2a10.pdf>. Acesso em: 06 nov. 2007.

\title{
Work accidents among healthcare professionals in a university hospital
}

\begin{abstract}
This research aimed to study the occurrence of work accidents involving healthcare professionals in a university hospital, determining those professional categories with greatest number of occurrences and the most critical sectors in the organization. The data analyzed from the work accidents register were: sex, age, profession, place of accident, type of accident, cause of lesion, affected area and number of hours between the beginning of the work journey to the accident. The documents were obtained through the Comissão Interna de Prevenção de Acidentes (CIPA), and data were analyzed with Qui-Square Test. Female individuals and the nursing team were the most affected, and most of the accidents involved biological material. There were significant occurrences between 0 and 4 hours after the beginning of the work journey. Information from this study should provide a basis for preventive measures among health and administrative professionals.
\end{abstract}

Keywords

Work accidents. Healthcare professionals. Hospital. 